\title{
SUPPLEMENTARY INFORAMTION
}

to

Trinuclear Palladium(II) Complex containing $S$-Benzyl ortho-aminothiophenol and 2-Thiolato-1,3-benzothiazole Ligands with a Central, Square Planar PdN Motif

Edward D. Cross, Kristen L. MacDonald, Robert McDonald ${ }^{\$}$, Matthias Bierenstiel*

Cape Breton University, Department of Chemistry, 1250 Grand Lake Road, Sydney, Nova Scotia, Canada, B1P 6L2

${ }^{\$}$ University of Alberta, X-Ray Crystallography Laboratory, Department of Chemistry, Edmonton, Alberta, Canada, T6G 262

*Corresponding Author:

Phone: $\quad 1$ (902) $563-1391$

Fax: $\quad 1(902) 563-1899$

Email: $\quad$ Matthias_Bierenstiel@cbu.ca 
Spectral data for NMR and MS of compound 2

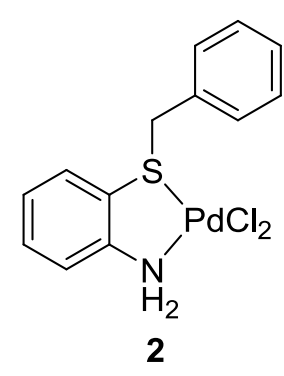


$\underline{\text { Supplementary Information }}$

${ }^{1} \mathrm{H}$ NMR spectrum (400.1 MHz, DMSO-d 6 )

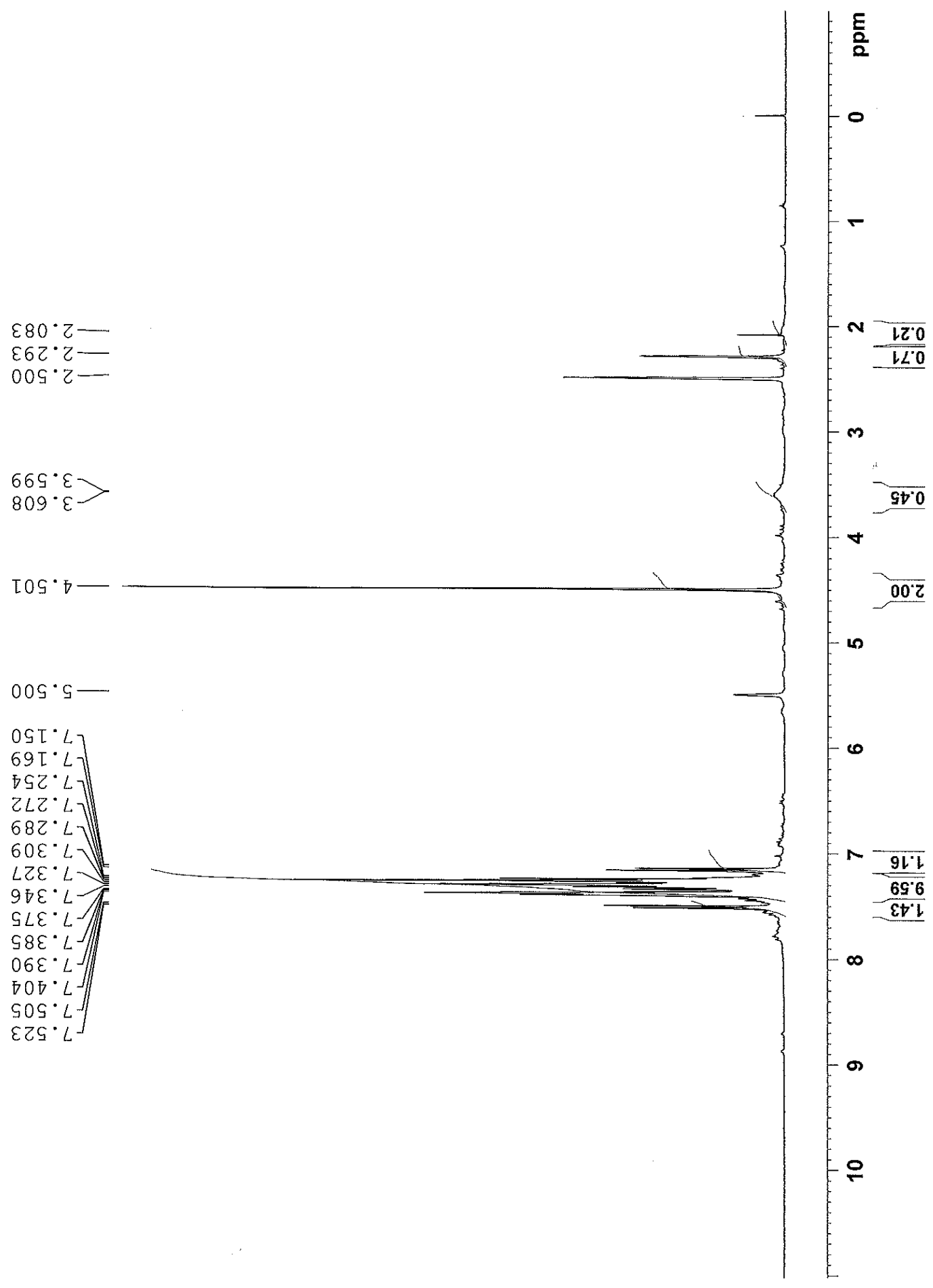


${ }^{1} \mathrm{H}$ NMR spectrum $(400.1 \mathrm{MHz}$, DMSO-d 6 ) - zoom
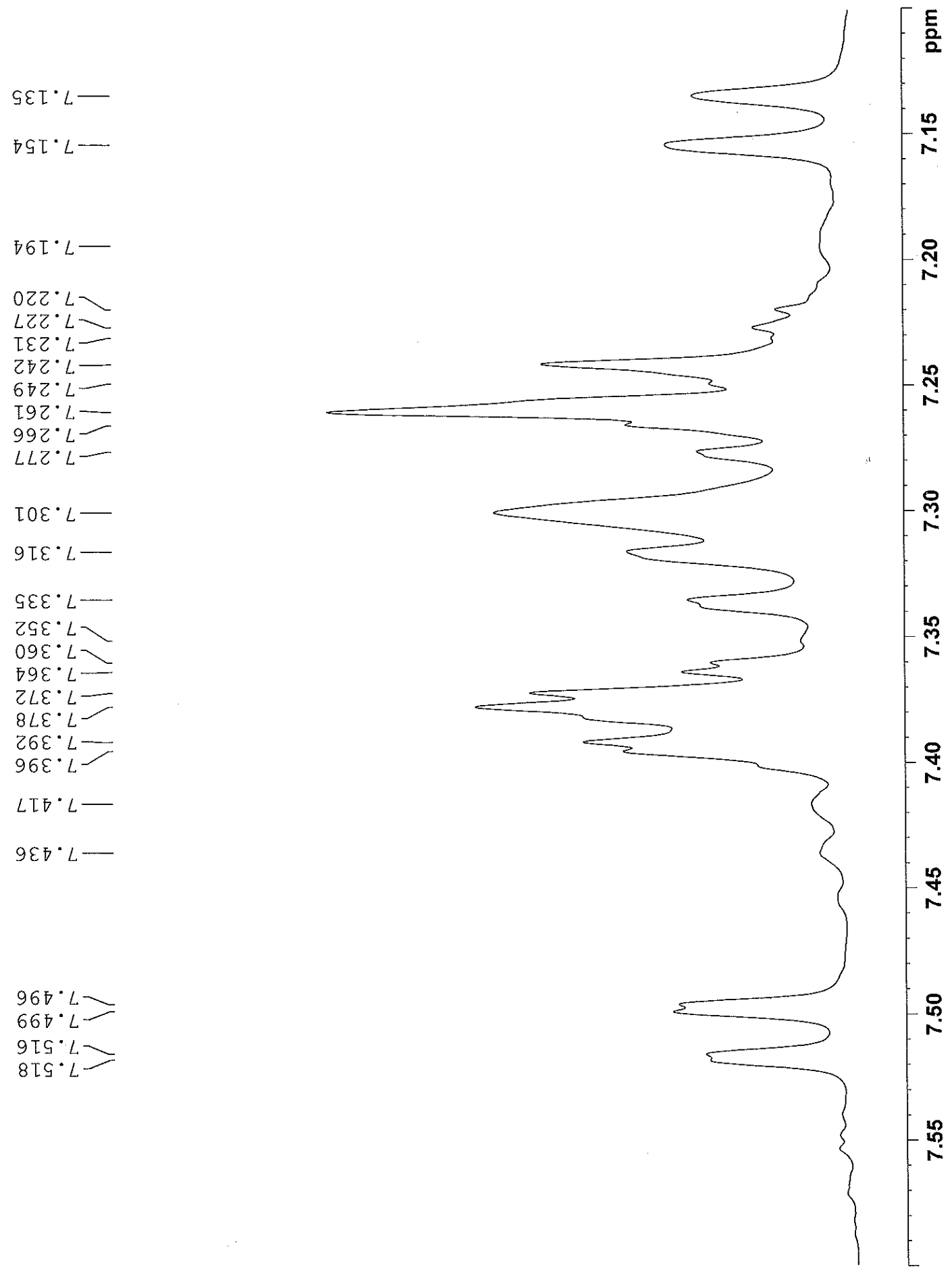
${ }^{13} \mathrm{C}$ NMR spectrum (100.7 MHz, DMSO-d ${ }_{6}$ )

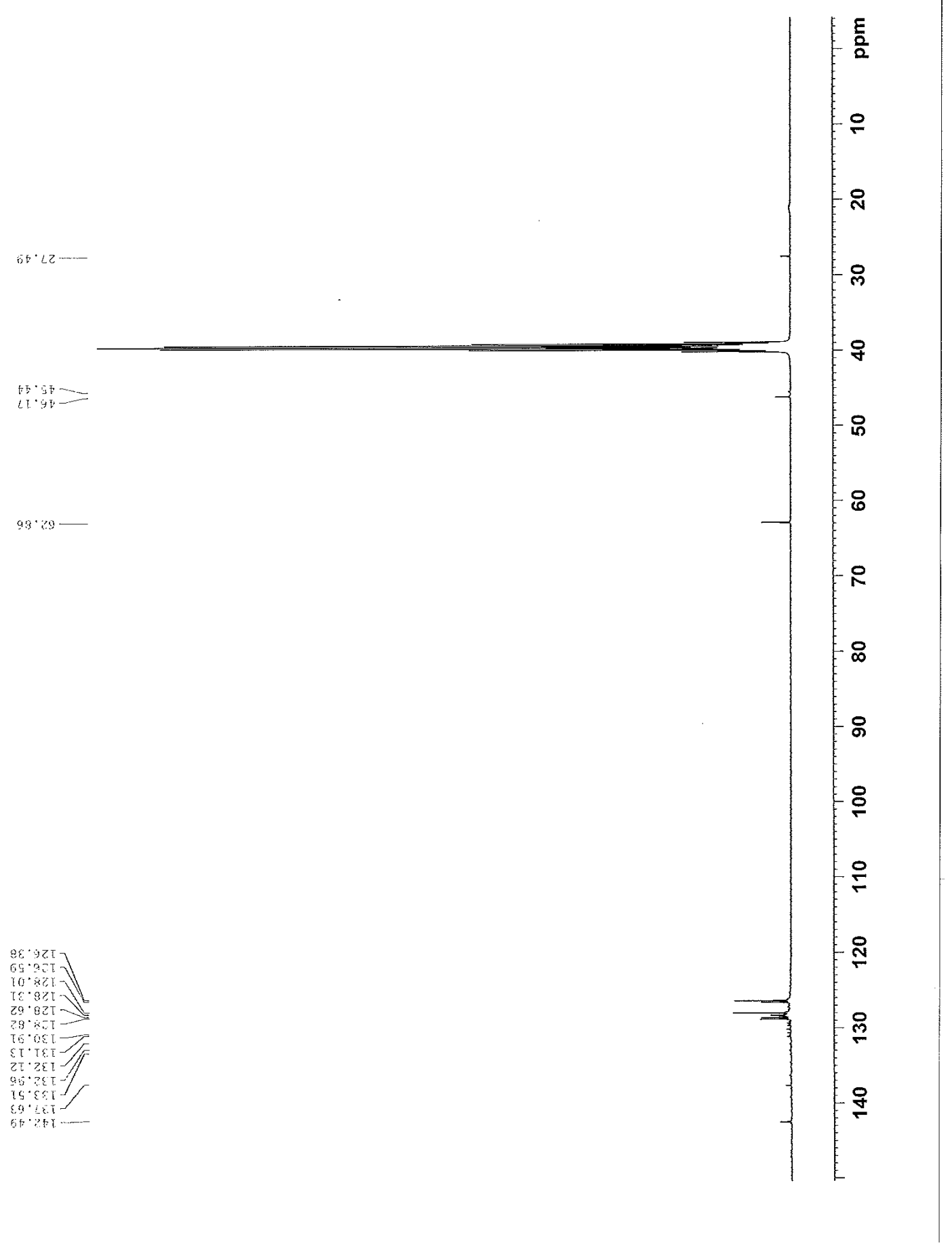


${ }^{13} \mathrm{C}$ NMR spectrum $\left(100.7 \mathrm{MHz}, \mathrm{DMSO}-\mathrm{d}_{6}\right)-$ zoom
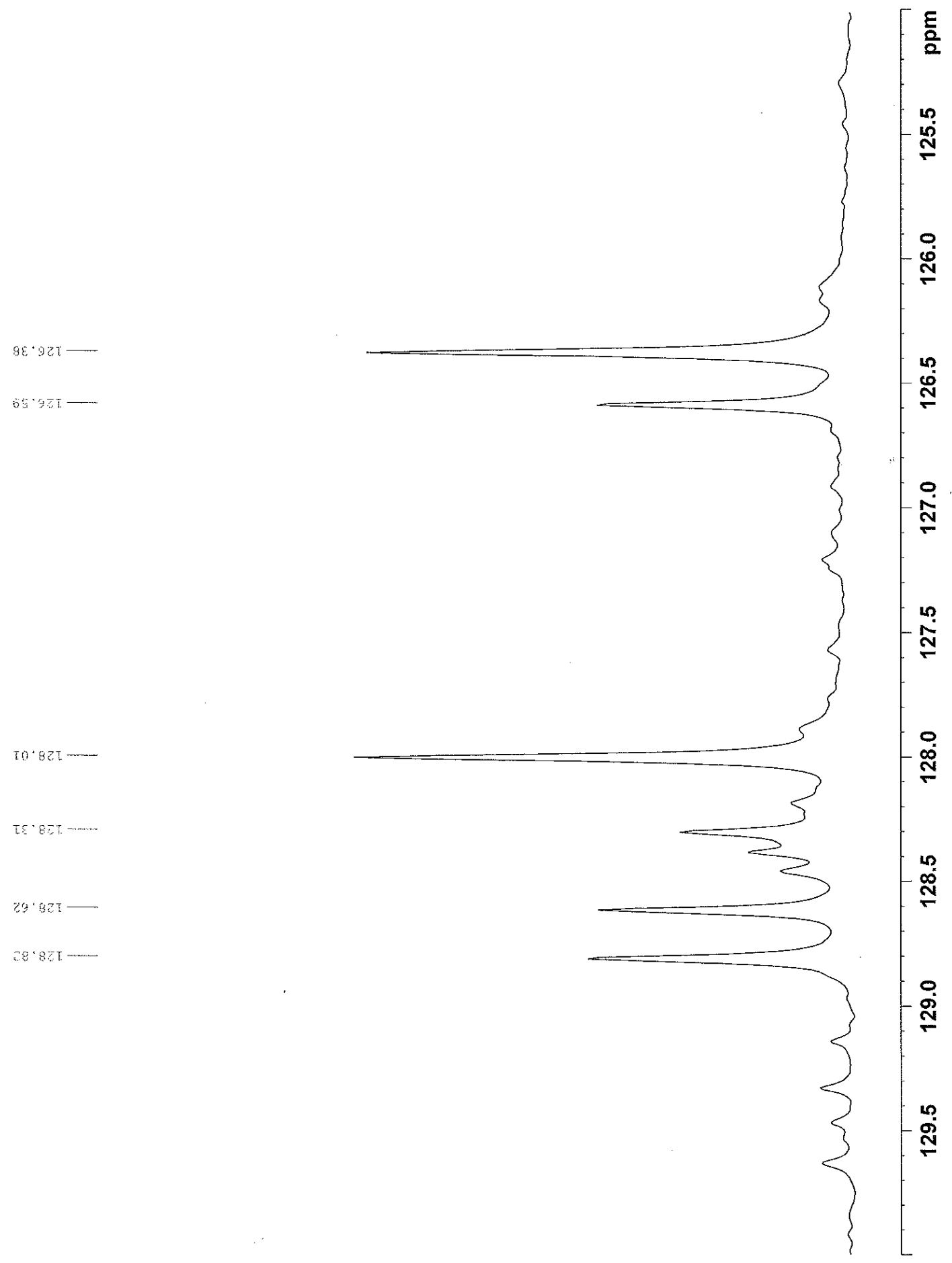
${ }^{13} \mathrm{C}$ NMR jmod spectrum (100.7 MHz, DMSO-d $\left.\mathrm{d}_{6}\right)$

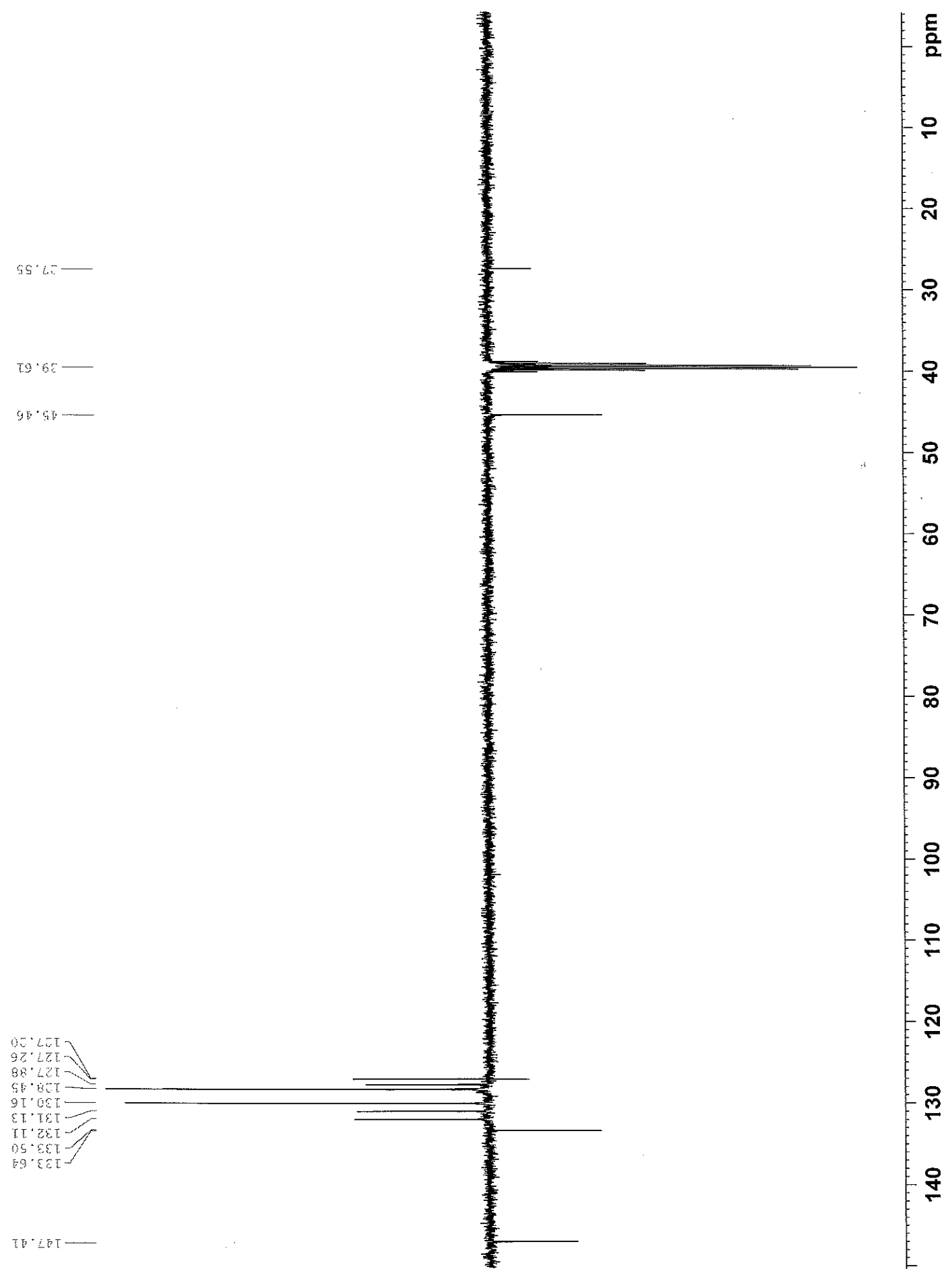


${ }^{13} \mathrm{C}$ NMR jmod spectrum (100.7 MHz, DMSO-d 6 - zoom
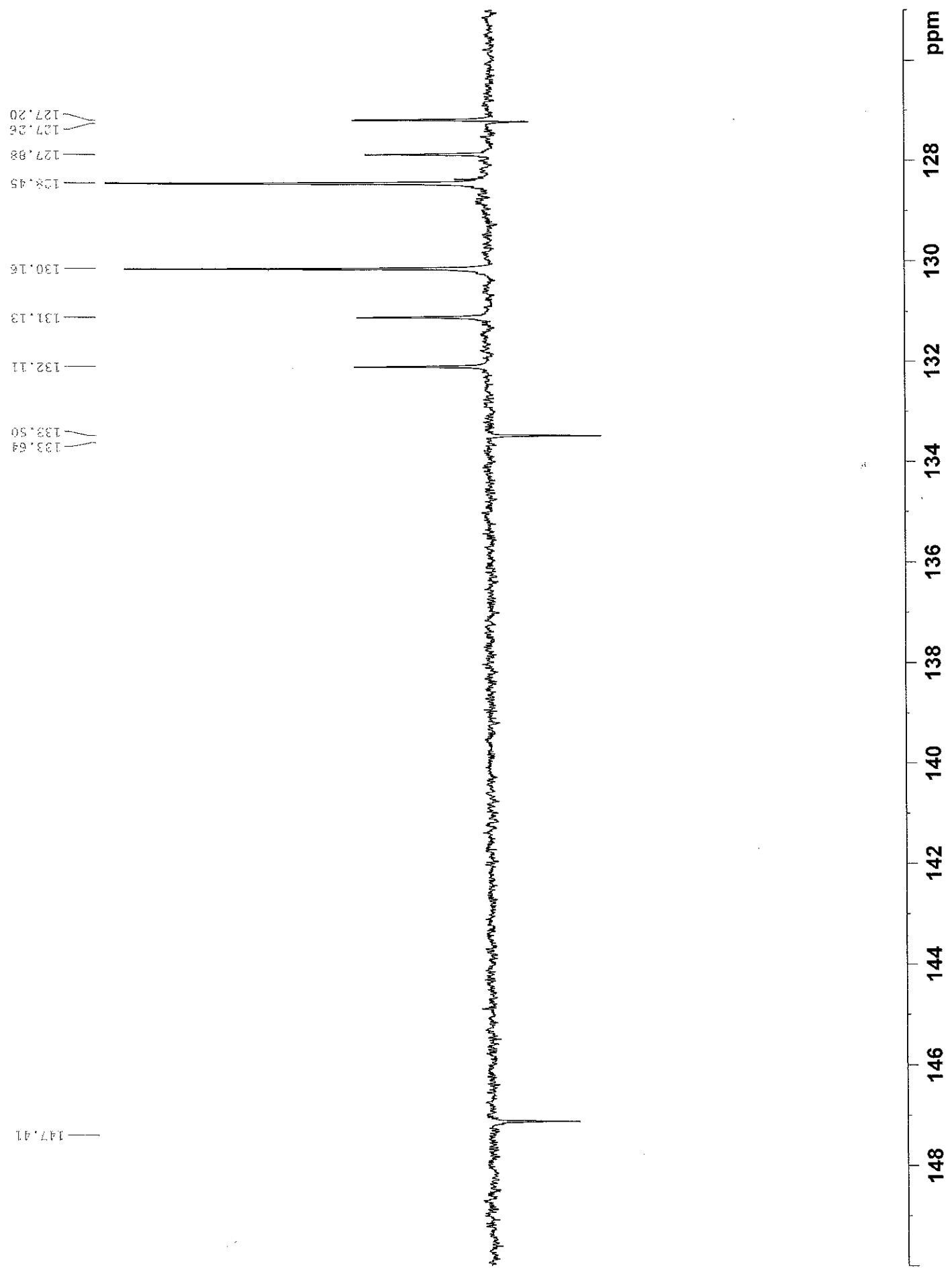
HSQC Spectrum (DMSO-d ${ }_{6}$ )

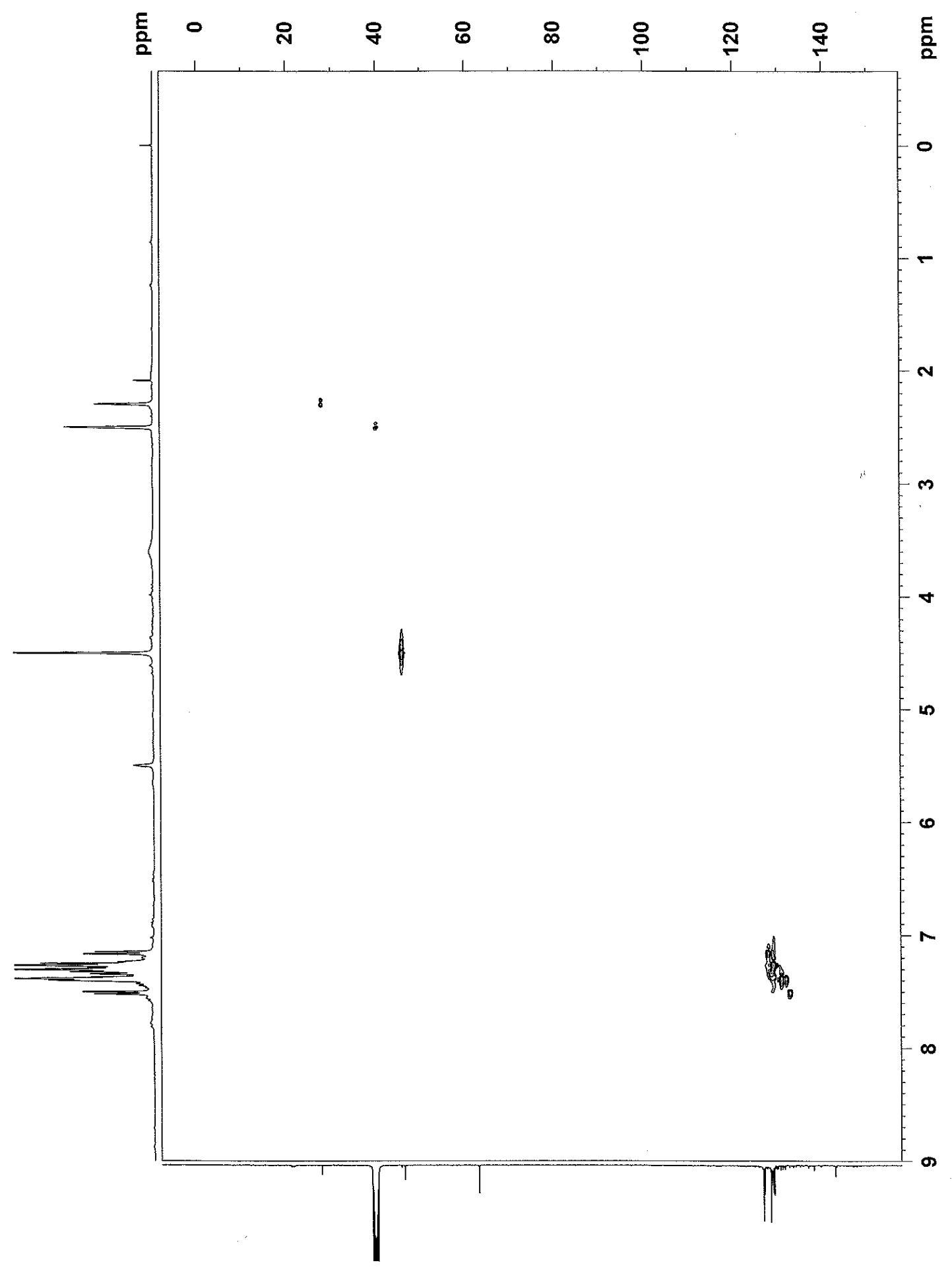




\section{UPLC-MSMS Information}

Column: Acquity UPLC BEH C18 1.7 $\mu \mathrm{m}, 2.1$ x $50 \mathrm{~mm}$

ESI Naformate_positive mode

Enhance accuirate mass range 50 to $1,200 \mathrm{~m} / \mathrm{z}$

Capillary $0.8 \mathrm{kV}$

Sampling cone $35.0 \mathrm{~V}$

Source T: $100^{\circ} \mathrm{C}$

Desolvation $\mathrm{T}: 550^{\circ} \mathrm{C}$

Cone gas flow: $50 \mathrm{~L} / \mathrm{h}$

Desolvation gas flow: $1,000 \mathrm{~L} / \mathrm{h}$

\section{LC trace}

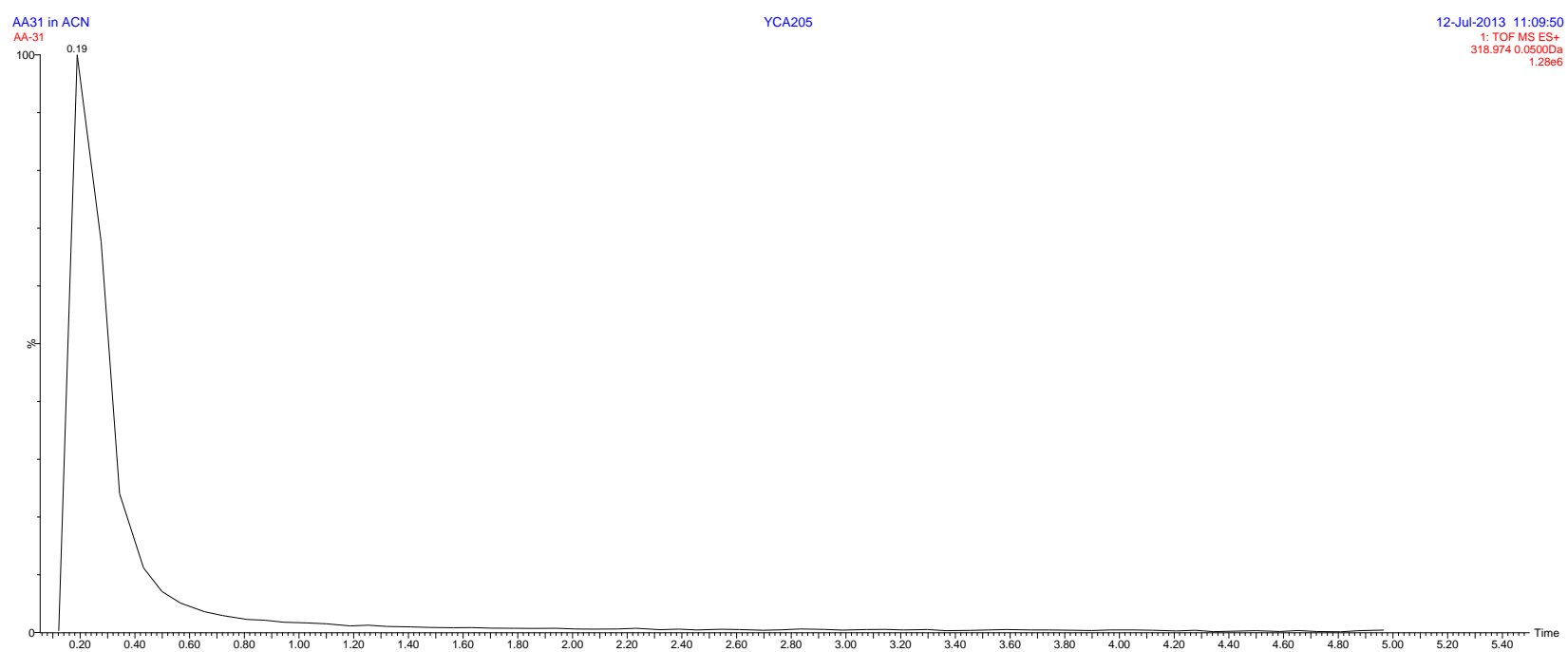


$\underline{\text { Supplementary Information }}$

\section{MS Spectrum}

Calculated (above) and experimental (below) MS spectrum of $319 \mathrm{~m} / \mathrm{z}$

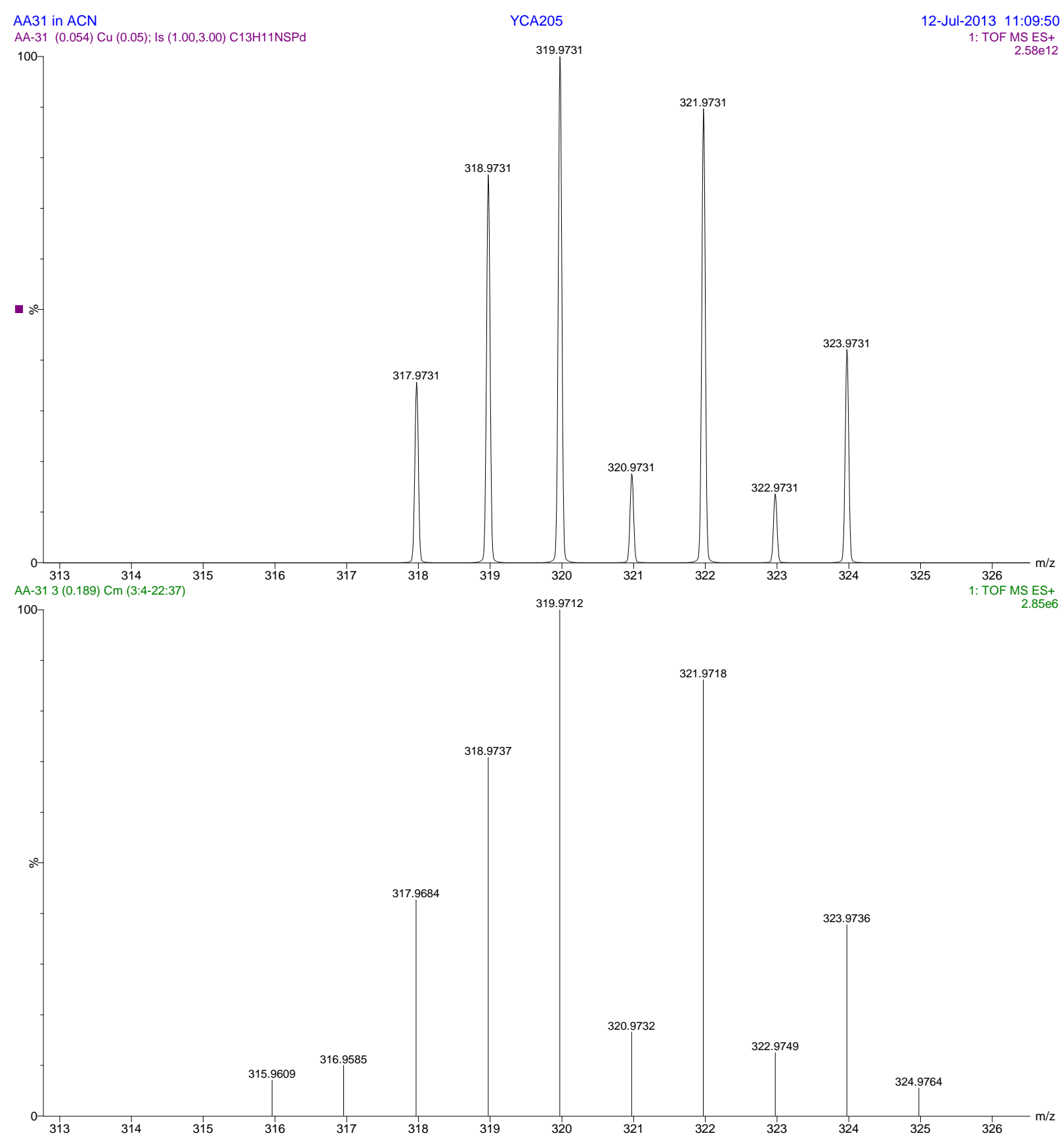

